\title{
Optimizing the Joint Replenishment and Delivery Scheduling Problem under Fuzzy Environment Using Inverse Weight Fuzzy Nonlinear Programming Method
}

\author{
Yu-Rong Zeng, ${ }^{1}$ Lin Wang, ${ }^{2}$ Xian-Hao Xu, ${ }^{2}$ and Qing-Liang Fu ${ }^{2}$ \\ ${ }^{1}$ School of Information Management, Hubei University of Economics, Wuhan 430205, China \\ ${ }^{2}$ School of Management, Huazhong University of Science \& Technology, Wuhan 430074, China \\ Correspondence should be addressed to Lin Wang; wanglin982@gmail.com
}

Received 25 February 2014; Accepted 21 June 2014; Published 22 July 2014

Academic Editor: Haydar Akca

Copyright ( $\odot 2014$ Yu-Rong Zeng et al. This is an open access article distributed under the Creative Commons Attribution License, which permits unrestricted use, distribution, and reproduction in any medium, provided the original work is properly cited.

In reality, decision-makers are always in front of imprecise and vague operational conditions. We propose a practical multiobjective joint replenishment and delivery scheduling (JRD) model with deterministic demand and fuzzy cost. This model minimizes the total cost defuzzified by the signed distance method and maximizes the credibility that the total cost does not exceed the budget level. Then, an inverse weight fuzzy nonlinear programming (IWFNLP) method is adopted to formulate the proposed model. This method embeds the idea of inverse weights into the Max-Min fuzzy model. Thirdly, the fuzzy simulation approach and differential evolution algorithm (DE) are utilized to solve this problem. Results show that solutions derived from the IWFNLP method satisfy the decision-maker's desirable achievement level of the cost objective and credibility objective. It is an effective decision tool since it can really reflect the relative importance of each fuzzy component. Our study also shows that the improved DE outperforms DE with a faster convergence speed.

\section{Introduction}

As a multi-item inventory problem, the joint replenishment problem (JRP) has been widely applied to lots of sizing problems in manufacturing applications (Hsu [1]; Goyal [2]; Wang et al. $[3,4])$. Beside the possible quantity discount, gathering several items on a single order to reduce the total of these fixed ordering costs is pretty reasonable (Kaspi and Rosenblatt [5]). The studies of the JRPs can be divided into two categories: (a) heuristics for the classic JRP under constant demand and special applications (Olsen [6]; Lee and Yao [7]; Porras and Dekker [8]) and (b) the JRPs under dynamic and/or stochastic demand. The extensive literature review is available in Khouja and Goyal [9] and Narayanan et al. [10].

Typically speaking, if the warehouse is assumed as the central of a supply chain for all the new JRPs, two extensions of JRP should be noted; one extension is in the supplying end and the other extension is in the selling end. For both of two extensions, the delivery considerations should be considered. Here we call them the joint replenishment and delivery scheduling (JRD) problems. In fact, most corporations with global purchasing have realized that considerable cost savings can be achieved by a JRD policy (Sindhuchao et al. [11]). The relatively scarce literature on the JRDs can be classified into two categories: (a) JRDs with deterministic demand, Chan et al. [12] addressed scheduling issues for multibuyer joint replenishments and Cha et al. [13] studied a JRD model of the one-warehouse and $n$-retailer system and developed a genetic algorithm (GA) and an improved heuristic named RAND to solve this problem and Moon et al. [14] provided joint replenishment and consolidated freight delivery policies with deterministic demand for a third party warehouse; (b) JRDs with stochastic demand, Wang et al. [15] proposed an effective and efficient differential evolution algorithm (DE) for the integrated stochastic JRD. Similar studies can be found in [16-18]. A limitation common in all the JRDs mentioned above is that all the key factors are assumed to be certain.

In reality, it is more realistic to handle imprecise values using the fuzzy theory for the JRD. In fact, decision-makers are always in front of imprecise and vague operational 
conditions (Pishvaee and Torabi [19]; Zeng et al. [20]). Uncertainties have been tackled in a lot of ways and fuzzy set theory has a long history for managing inventory (Chiang et al. [21]; Kacprzyk and Stanieski [22]; Wang et al. [23, 24]; Wee et al. [25]). Generally, the imprecision may originate from two aspects in the JRD modeling: (1) the imprecise specification of objectives, for example, a decision-maker may have to face vague goals such as "this total cost should be around \$20000"; (2) the imprecise specification of related parameters, an important task involved in the fuzzy JRD model is to predict parameters' values such as the holding cost and major ordering cost. However, due to the nonavailability of sufficient and precise input data, the precise predicted values cannot be obtained easily; just an "approximate" value may be ascertained, while fuzzy numbers can efficiently model the imprecise values.

At present, there are two common approaches to handle fuzzy parameters. (1) Defuzzification. As a favorite approach in many inventory modeling for its simplicity, defuzzification can easily transfer fuzziness to be explicit without complex analysis (Roy et al. [26]). Handfield et al. [27] defuzzified a $(Q, r)$ model based on fuzzy-set representations of various sources of uncertainly in the supply chain. (2) Fuzzy dependent-chance programming (DCP). Fuzzy DCP maximizes the credibility of event such that the total cost in the planning periods does not exceed a certain budget level (Liu [28]). Wang et al. [29] studied a novel JRP model based on DCP with fuzzy minor replenishment cost and fuzzy inventory holding cost. Similar paper can be found in Peng and Zhou [30]. For the JRP decision, managers also hope that the total cost does not exceed the budget level, especially when enterprises have much cash flow pressure. In this situation, a fuzzy DCP model is always regarded as a good choice.

However, no study has simultaneously considered total cost and credibility of the total cost does not exceed the budget level as performance criterions for the JRD with fuzzy cost. This unexplored area is important and interesting since it integrates into a single model two main decision criterions: total cost and credibility. The aim of this paper is to develop a practical multiobjective JRD (M-JRD) model with deterministic demand and fuzzy cost firstly. Moreover, an inverse weight fuzzy nonlinear programming (IWFNLP) adopted by [25] is applied to the M-JRD to satisfy the decision-maker's desirable achievement level of each fuzzy component. At last, novel hybrid algorithms are provided using the fuzzy simulation approach (FSA) and DE for this model handled by IWFNLP method. Results of examples show solutions derived from the IWFNLP method satisfy the decisionmaker's desirable achievement level of the cost and credibility objective. It is an effective decision tool since it can really reflect the relative importance of each fuzzy component.

The rest of this paper is organized as follows. The multiobjective JRD model is given in Section 2. In Section 3, the fuzzy simulation for credibility calculation and DE for searching for an optimum solution are introduced. Section 4 is numerical examples and analysis. Section 5 contains conclusions and future research directions.

\section{Mathematical Model and Analysis}

\subsection{Assumptions and Formulation of Fuzzy JRD Model}

(1) Assumptions and Notations. Lu and Posner [31] addressed the problem of supplying multiple retail outlets with constant and continuous demand from a single warehouse. Each retailer sells one product, and other retailers sell the same product. All demands must be met without shortages or backlogging. Orders placed by a retailer will result in demand generated at the warehouse. The objective is to minimize the average total cost. This one warehouse, $n$-retailer JRD problem has received considerable attention from researchers $[13,14]$. In this study, we focus on the JRD model under fuzzy environment. The following assumptions are similar with Lu and Posner [31] and Cha et al. [13]. (1) All parameters including demand rates and costs (except warehouse's major ordering cost) are known and constant; (2) replenishment is instantaneous; (3) replenishment lead time is constant.

As reported in Wang et al. [23], an interesting real problem is when human originated data like ordering cost are not precisely known but subjectively estimated or linguistically expressed because of the lack of the accurate history data. Suppose that the major ordering cost $(S)$ is not precisely known. For example, let the linguistic estimates of $S$ be as follows. "The major ordering cost is about 40 dollars per order, but not less than 20 dollars per order nor more than 60 dollars per order." In reality, it is very hard to obtain the precise cost. So fuzzy variables are also utilized to handle the JRD problem under uncertainty. In this study, warehouse's major ordering cost is treated as a fuzzy number. Our work introduces fuzziness into the JRD which makes it become more practical. Due to the JRD's difficult mathematical properties, we just suppose the major ordering cost as a fuzzy number to make the study become more possible because two different objectives are considered simultaneously.

As presented in Cha et al. [13], the warehouse will deliver to an individual retailer after it replenishes goods jointly from the suppliers by taking into account the demand of each item at the retailer. The warehouse that has a lot of associated retailers can obtain significant cost savings (logistics cost and ordering cost) by replenishing jointly. The reduction effects will be higher if materials are imported from overseas with the high procurement cost. Referring to [13], the JRD model in this study can be described in Figure 1. Note the warehouse's major ordering cost is a fuzzy number.

In order to discuss the JRD problem, the following notations are defined:

$n$ : number of items;

$i$ : index of item, $i=1,2, \ldots, n$;

$D_{i}$ : demand rate of item $i$;

$\widetilde{S}$ : warehouse's major ordering cost, a triangle fuzzy number;

$s_{i}^{W}$ : warehouse's minor ordering cost of item $i$; 


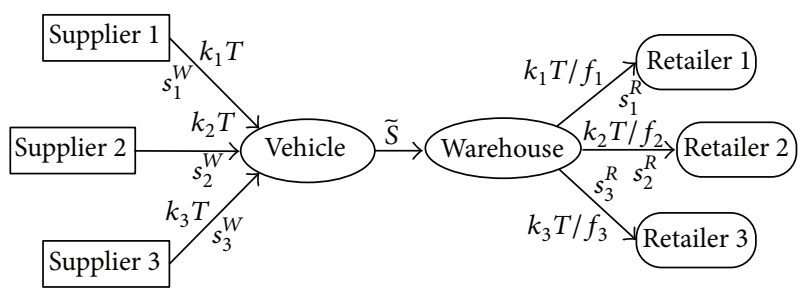

FIgURE 1: The JRD model (source: Cha et al. [13]).

$h_{i}^{W}$ : warehouse's inventory holding cost of item $i$ per unit time;

$s_{i}^{R}$ : warehouse's outbound transportation cost of item $i$;

$h_{i}^{R}$ : retailer's inventory holding cost of item $i$ per unit per time;

$T$ : warehouse's basic cycle time (decision variable);

$f_{i}$ : integer number that decides the outbound schedule of item $i$ (decision variable);

$k_{i}$ : integer number that decides the replenishment schedule of item $i$ (decision variable), it means the replenishment cycle of item $i$ is $k_{i} T$.

(2) Formulation with Fuzzy Major Ordering Cost. Similar to [13], we also discuss $n$ different types of productions and assume that all productions can be stored at the warehouse. Further, we assume that item $i$ is stored and sold only by retailer $i$. The warehouse replenishes item $i$ at every integer multiple $\left(k_{i}\right)$ of the basic cycle time $(T)$ and delivers it to retailer $i$.

Procedures to find optimal policies are very difficult. There are no known good approaches for solving this problem in time polynomial in the number of retailers ( $\mathrm{Lu}$ and Posner [31]). Most scholars have concentrated on developing good heuristics for special policies. In this study, a popular stationary policy used in [13] is also adopted that a warehouse delivers item $i$ to retailer $i$ at the same time interval with a fixed order quantity.

The total cost (TC) is composed of the sum of the major ordering cost, minor ordering cost, inventory holding cost, and outbound transportation cost of a warehouse as well as the total of the inventory holding costs of retailers. According to the above definitions, the total relevant fuzzy cost per unit time to be minimized is given by

$$
\begin{aligned}
\widetilde{C}\left(T, k_{i}^{\prime} s, f_{i}^{\prime} s\right)= & \frac{\widetilde{S}+\sum_{i=1}^{n}\left(s_{i}^{W} / k_{i}\right)}{T}+\sum_{i=1}^{n} \frac{\left(f_{i}-1\right) k_{i} T D_{i} h_{i}^{W}}{2 f_{i}} \\
& +\sum_{i=1}^{n} \frac{f_{i} s_{i}^{R}}{k_{i} T}+\sum_{i=1}^{n} \frac{k_{i} T D_{i} h_{i}^{R}}{2 f_{i}} .
\end{aligned}
$$

\subsection{Proposed Fuzzy Multiobjective JRD (M-JRD) Model and Analysis}

\subsubsection{Defuzzified Total Cost by Signed Distance}

(1) Defuzzified TC. The signed distance method is simple and easy to handle, and this is why the extension principle and centroid method are not applied to this fuzzy JRD model. It is difficult for the extension principle and centroid to obtain the estimated TC in fuzzy sense. Moreover, Chiang et al. [21] found there was not a significant difference between signed distance method and the extension principle or centroid method. So the signed distance method is used to obtain the TC in fuzzy sense.

From (1), we can obtain the following equations:

$$
\begin{aligned}
\bar{C}= & \frac{\bar{S}+\sum_{i=1}^{n}\left(s_{i}^{W} / k_{i}\right)}{T}+\sum_{i=1}^{n} \frac{\left(f_{i}-1\right) k_{i} T D_{i} h_{i}^{W}}{2 f_{i}} \\
& +\sum_{i=1}^{n} \frac{f_{i} s_{i}^{R}}{k_{i} T}+\sum_{i=1}^{n} \frac{k_{i} T D_{i} h_{i}^{R}}{2 f_{i}}, \\
C= & \frac{S+\sum_{i=1}^{n}\left(s_{i}^{W} / k_{i}\right)}{T}+\sum_{i=1}^{n} \frac{\left(f_{i}-1\right) k_{i} T D_{i} h_{i}^{W}}{2 f_{i}} \\
& +\sum_{i=1}^{n} \frac{f_{i} s_{i}^{R}}{k_{i} T}+\sum_{i=1}^{n} \frac{k_{i} T D_{i} h_{i}^{R}}{2 f_{i}}, \\
\underline{C}= & \frac{S}{+\sum_{i=1}^{n}\left(s_{i}^{W} / k_{i}\right)}+\sum_{i=1}^{n} \frac{\left(f_{i}-1\right) k_{i} T D_{i} h_{i}^{W}}{2 f_{i}} \\
& +\sum_{i=1}^{n} \frac{f_{i} s_{i}^{R}}{k_{i} T}+\sum_{i=1}^{n} \frac{k_{i} T D_{i} h_{i}^{R}}{2 f_{i}} .
\end{aligned}
$$

From (2), the defuzzified TC to be minimized using signed distance can be obtained as

$$
\begin{aligned}
d_{0}(\widetilde{C}, 0)= & \left(\frac{S}{2 T}+\frac{\bar{S}}{4 T}+\frac{\underline{S}}{4 T}\right)+\frac{\sum_{i=1}^{n}\left(s_{i}^{W} / k_{i}\right)}{T} \\
& +\sum_{i=1}^{n} \frac{\left(f_{i}-1\right) k_{i} T D_{i} h_{i}^{W}}{2 f_{i}}+\sum_{i=1}^{n} \frac{f_{i} s_{i}^{R}}{k_{i} T}+\sum_{i=1}^{n} \frac{k_{i} T D_{i} h_{i}^{R}}{2 f_{i}} .
\end{aligned}
$$

2.2.2. Credibility Object Based on DCP. Generally speaking, DCP is related to maximizing some chance functions events in an uncertain environment (Liu [28]). As a widely used type of stochastic programming, DCP has been extended to the area of fuzzy programming ( $R$. Wang and L. Wang [32]; Wang et al. [33]). In practice, the goal of JRD policy cannot be confirmed exactly due to inevitable uncertainty. Hence, a realistic approach for decision makers (DMs) may be to maximize the credibility of achieving the optimization goals. Sometimes, DMs are not concerned with minimizing the total cost but hope that TC does not exceed the budget level $\mathrm{TC}_{-}$, especially under much cash flow pressure. In this 
situation, a natural idea is to maximize the credibility of the event such that the total cost is less than or equal toTC_. So an objective function can be written as

$$
\max \operatorname{Cr}\left\{\widetilde{C}\left(T, k_{i}^{\prime} s, f_{i}^{\prime} s\right) \leq \mathrm{TC}_{-}\right\} .
$$

2.2.3. Proposed Fuzzy Multiobjective JRD (M-JRD) Model. Due to the uncertainty of the decision-making, it is quite natural to assume two main goals: (1) nonrigid total cost goal; (2) credibility goal to assure the safety of cash flow. These goals represent different attitudes of managers for handling the inevitable uncertainty. In reality, it is not surprising that managers have their own opinions on the goal under uncertainty. For the fuzzy objectives, the M-JRD model can be described as

$$
\begin{array}{ll}
\widetilde{\text { Min }} & d_{0}(\widetilde{C}, 0) \\
\widetilde{\operatorname{Max}} & \operatorname{Cr}\left\{\widetilde{C}\left(T, k_{i}^{\prime} s, f_{i}^{\prime} s\right) \leq \mathrm{TC}_{-}\right\} \\
\text {subject to } & T>0 \\
& k_{i} \geq 1 \text { : integer, } \quad i=1, \ldots, n \\
& f_{i} \geq 1 \text { : integer, } \quad i=1, \ldots, n,
\end{array}
$$

where the wavy bar " $\sim$ " denotes the fuzziness of the two objectives goal.

To describe the fuzzy objectives in (13), the decision-maker may subjectively define the acceptable interval $\left[d_{0}(\widetilde{C}, 0)^{0}, d_{0}(\widetilde{C}, 0)^{1}\right]$ for the defuzzification objective and $\left[C r\{\cdot\}^{0}, C r\{\cdot\}^{1}\right]$ for the credibility objective, where $\cdot$ denotes $\widetilde{C}\left(T, k_{i}^{\prime} s, f_{i}^{\prime} s\right) \leq \mathrm{TC}_{\text {. }}$.

Alternatively, the following four cases can be solved to confirm the values of $d_{0}(\widetilde{C}, 0)^{0}, d_{0}(\widetilde{C}, 0)^{1}, \operatorname{Cr}\{\cdot\}^{0}$, and $\operatorname{Cr}\{\cdot\}^{1}$, respectively.

(1) The first one minimizes

$$
\begin{aligned}
& \frac{\underline{S}+\sum_{i=1}^{n}\left(s_{i}^{W} / k_{i}\right)}{T}+\sum_{i=1}^{n} \frac{\left(f_{i}-1\right) k_{i} T D_{i} h_{i}^{W}}{2 f_{i}} \\
& +\sum_{i=1}^{n} \frac{f_{i} s_{i}^{R}}{k_{i} T}+\sum_{i=1}^{n} \frac{k_{i} T D_{i} h_{i}^{R}}{2 f_{i}},
\end{aligned}
$$

and the result is set as $d_{0}(\widetilde{C}, 0)^{0}$.

(2) The second one minimizes

$$
\begin{aligned}
& \frac{\bar{S}+\sum_{i=1}^{n}\left(s_{i}^{W} / k_{i}\right)}{T}+\sum_{i=1}^{n} \frac{\left(f_{i}-1\right) k_{i} T D_{i} h_{i}^{W}}{2 f_{i}} \\
& +\sum_{i=1}^{n} \frac{f_{i} s_{i}^{R}}{k_{i} T}+\sum_{i=1}^{n} \frac{k_{i} T D_{i} h_{i}^{R}}{2 f_{i}},
\end{aligned}
$$

and the result is set as $d_{0}(\widetilde{C}, 0)^{1}$.

(3) The third one maximizes $\operatorname{Cr}\left\{\widetilde{C}\left(T, k_{i}^{\prime} s, f_{i}^{\prime} s\right) \leq\right.$ $\left.d_{0}(\widetilde{C}, 0)^{0}\right\}$, and the result is set as $\operatorname{Cr}\{\cdot\}^{0}$.
(4) The forth one maximizes $\operatorname{Cr}\left\{\widetilde{C}\left(T, k_{i}^{\prime} s, f_{i}^{\prime} s\right) \leq\right.$ $\left.d_{0}(\widetilde{C}, 0)^{1}\right\}$, and the result is set as $\operatorname{Cr}\{\cdot\}^{1}$.

Then, the membership function is used to describe the attainable degree of the two objectives. In this study, the linear membership functions $\mu_{\widetilde{d}_{0}(\widetilde{C}, 0)}\left(d_{0}(\widetilde{C}, 0)\right), \mu_{\widetilde{C} r\{\}}(\operatorname{Cr}\{\cdot\})$ are used, where

$$
\begin{aligned}
& \mu_{\widetilde{d}_{0}(\widetilde{C}, 0)}\left(d_{0}(\widetilde{C}, 0)\right) \\
& =\left\{\begin{array}{lr}
1 & \text { for } d_{0}(\widetilde{C}, 0)<d_{0}(\widetilde{C}, 0)^{0} \\
\frac{d_{0}(\widetilde{C}, 0)^{1}-d_{0}(\widetilde{C}, 0)}{d_{0}(\widetilde{C}, 0)^{1}-d_{0}(\widetilde{C}, 0)^{0}} & \text { for } d_{0}(\widetilde{C}, 0)^{0} \leq d_{0}(\widetilde{C}, 0) \\
& \leq d_{0}(\widetilde{C}, 0)^{1} \\
0 & \text { for } d_{0}(\widetilde{C}, 0)>d_{0}(\widetilde{C}, 0)^{1},
\end{array}\right.
\end{aligned}
$$

$$
\begin{array}{ll}
\mu_{\tilde{C} r\{\cdot\}}(\operatorname{Cr}\{\cdot\}) & \\
= & \begin{cases}0 & \text { for } \operatorname{Cr}\{\cdot\}<\operatorname{Cr}\{\cdot\}^{0} \\
\frac{\operatorname{Cr}\{\cdot\}^{1}-\operatorname{Cr}\{\cdot\}^{0}}{1} & \text { for } \operatorname{Cr}\{\cdot\}^{0} \leq \operatorname{Cr}\{\cdot\} \leq \operatorname{Cr}\{\cdot\}^{1} \\
1 & \text { for } \operatorname{Cr}\{\cdot\}<\operatorname{Cr}\{\cdot\}^{1} .\end{cases}
\end{array}
$$

The pictorial representations of these membership functions are given in Figure 2.

\subsection{Mathematical Analysis Using IWFNLP Method}

2.3.1. Formulation Using Traditional Fuzzy Additive Goal Programming. In order to specify imprecise aspiration levels of the goals under fuzzy environment, Narasimhan [34] had firstly developed fuzzy goal programming (FGP) using membership functions. Chen and Tsai [35] reformulated the fuzzy additive goal programming (FAGP) by incorporating different important and preemptive priorities of fuzzy goals. In contrast to other methods, the FAGP allows managers to determine a desirable achievement degree for each fuzzy goal to reflect explicitly the relative importance of these goals. In order to better understand the following proposed $\mathrm{M}$ JRD model using IWFNLP, we give the formulation using traditional FAGP as follows:

$$
\begin{array}{ll}
\operatorname{Max} & w_{1} \mu_{1}+w_{2} \mu_{2} \\
\text { subject to } & \frac{d_{0}(\widetilde{C}, 0)^{1}-d_{0}(\widetilde{C}, 0)}{d_{0}(\widetilde{C}, 0)^{1}-d_{0}(\widetilde{C}, 0)^{0}}=\mu_{1} \\
& \frac{C r\{\cdot\}-C r\{\cdot\}^{0}}{C r\{\cdot\}^{1}-C r\{\cdot\}^{0}}=\mu_{2} \\
T>0 & k_{i} \geq 1 \text { : integer, } \quad i=1, \ldots, n \\
& f_{i} \geq 1 \text { : integer, } \quad i=1, \ldots, n,
\end{array}
$$




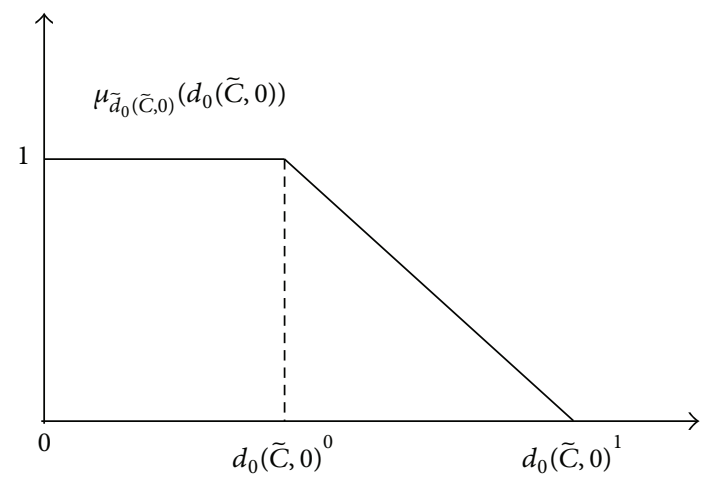

(a)

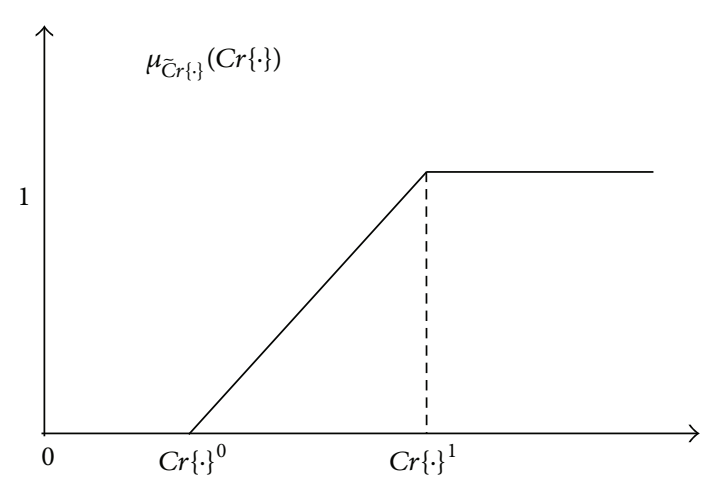

(b)

Figure 2: Membership functions for fuzzy total cost and credibility objectives.

where $w_{1}$ and $w_{2}$ are weights for the defuzzification objective and credibility objective, respectively, and $w_{1}+w_{2}=1\left(w_{1}>\right.$ $\left.0 ; w_{2}>0\right)$. These weights reflect the relative importance of each goal in the decision model.

\subsubsection{Formulation Using IWFNLP Approach}

(1) Rationales of Using IWFNLP. Although the FAGP can be applied to handle the fuzzy decision problem, Wee et al. [25] found that the ratio of the achievement levels $\left(\mu_{1}, \mu_{2}\right)$ obtained by this approach cannot really reflect the ratio of the weights. To overcome the shortages of the FAGP method, a novel IWFNLP method was adopted. This method embeds the idea of inverse weights into the Max-Min fuzzy model. Results of a lot of experiments show IWFNLP method can give solutions in which the ratio of the fuzzy components achievement levels is as close to the ratio of the assigned weights as possible. So the IWFNLP is also adapted to handle the M-JRD. The effectiveness of this method is further verified in Section 4.

(2) Formulation. For the weights $w_{r}(r=1,2, \ldots, m)$, one-toone transformation from the weight $w_{r}$ to the inverse weight $\mathrm{IW}_{r}$ is defined as

$$
\mathrm{IW}_{r}=\frac{1 / w_{r}}{\sum_{l 1=1}^{m} 1 / w_{l 1}}=\frac{1}{w_{r} \sum_{l 1=1}^{m} 1 / w_{l 1}}
$$

In this study, $m=2$. So the fuzzy model is formulated as

$$
\begin{array}{ll}
\operatorname{Max} & \lambda \\
\text { subject to } & \operatorname{IW}_{1}\left(\frac{d_{0}(\widetilde{C}, 0)^{1}-d_{0}(\widetilde{C}, 0)}{d_{0}(\widetilde{C}, 0)^{1}-d_{0}(\widetilde{C}, 0)^{0}}\right) \geq \lambda \\
& \operatorname{IW}_{2}\left(\frac{\operatorname{Cr}\{\cdot\}-\operatorname{Cr}\{\cdot\}^{0}}{\operatorname{Cr}\{\cdot\}^{1}-\operatorname{Cr}\{\cdot\}^{0}}\right) \geq \lambda \\
& T>0
\end{array}
$$

$$
\begin{aligned}
& k_{i} \geq 1: \text { integer }, \quad i=1, \ldots, n \\
& f_{i} \geq 1 \text { : integer, } \quad i=1, \ldots, n \\
& \lambda \geq 0,
\end{aligned}
$$

where $\mathrm{IW}_{r}=1 /\left(w_{r} \sum_{l 1=1}^{2} 1 / w_{l 1}\right), r=1,2$.

According to the definition of inverse weight, (12) is equivalent to

$$
\begin{array}{ll}
\operatorname{Max} & \lambda \\
\text { subject to } & \frac{d_{0}(\widetilde{C}, 0)^{1}-d_{0}(\widetilde{C}, 0)}{d_{0}(\widetilde{C}, 0)^{1}-d_{0}(\widetilde{C}, 0)^{0}} \geq w_{1} \lambda\left(\sum_{l=1}^{2} \frac{1}{w_{l}}\right) \\
& \frac{\operatorname{Cr}\{\cdot\}-\operatorname{Cr}\{\cdot\}^{0}}{\operatorname{Cr}\{\cdot\}^{1}-\operatorname{Cr}\{\cdot\}^{0}} \geq w_{2} \lambda\left(\sum_{l=1}^{2} \frac{1}{w_{l}}\right) \\
& T>0 \\
& k_{i} \geq 1 \text { : integer, } \quad i=1, \ldots, n \\
& f_{i} \geq 1 \text { : integer, } \quad i=1, \ldots, n \\
& \lambda \geq 0 .
\end{array}
$$

\section{The Proposed Algorithm for Fuzzy M-JRD Using FSA and DE}

\subsection{Outlet of the Proposed Algorithm}

(1) The FSA Is Utilized to Solve the Fuzzy DCP. Usually, it is hard to obtain credibility value with an analytical method. Wang et al. [33] designed a FSA to calculate the credibility value for fixed decision variables. The advantage of the FSA is that it can calculate the credibility whether the membership functions of the fuzzy numbers are simple or not and whether several fuzzy numbers are involved or not. In fact, analytical methods usually can also be utilized for the same target. However, it can work only when member functions are simple, for example, linear functions and triangular functions. 
Moreover, analytical methods do not work well when too many fuzzy numbers are involved. But the FSA does not subject to such a restraint. So the FSA is adopted in this study.

(2) DE Is Adopted to Find the Solution Quickly. When the objective functions to be optimized are multimodal or the search spaces are particularly irregular, intelligent algorithms should be designed to solve the fuzzy DCP models. Moreover, optimization algorithms need to be highly robust in order to avoid getting stuck at the local optimal solution. Among these algorithms, genetic algorithm (GA) has been proved to be effective for the DCP models (Ke and Liu [36]). However, the GA displays inherent difficulties in performing local search for some applications because of the difficulty of the selection of the suitable probability of crossover and mutation. So it is important to find a novel algorithm to handle the fuzzy DCP more effectively.

$\mathrm{DE}$ is one of the best evolution algorithms (EAs) for solving nonlinear, nondifferentiable, and multimodal optimization problems (Storn and Price [37]). Due to its simple structure, easy implementation, quick convergence, and robustness, DE has been applied to a variety of fields [38-43]. Wang et al. [4] found that the basic DE is a good candidate for the similar JRPs. But, the effectiveness of DEs for the fuzzy JRD should be studied further because of the difficult mathematical properties.

DE has a good performance in convergence speed, but the faster convergence may cause the diversity of population to descend quickly during the solution process [42]. A good trade-off between convergence and diversity should be designed. Zou et al. [44] developed an improved DE (IDE) with a modified mutation factor according to the objective function values in mutation steps and adjusted crossover factor in terms of iteration number in crossover step. The IDE can not only diversify candidate solutions, but also increase the convergence rate. So the IDE is utilized in case of DE having unsatisfactory performance.

3.2. Fuzzy Simulation Approach for Solving DCP-Based Model. Usually, it is difficult to compute $\mathrm{Cr}\{\cdot\}$ with an analytic method. Instead, we use a FSA to obtain the value of $\operatorname{Cr}\{\cdot\}$ for a fixed $\left(T, k_{i}^{\prime} s, f_{i}^{\prime} s\right)$. According to [33], a classic fuzzy simulation approach can be adopted as follows.

Step 1. Set $e_{1}=0, e_{2}=0$, and $z=1$. Set the maximum iteration times $N_{1}$.

Step 2. Uniformly generate a sequence $\theta_{1 z}$ from $\Theta$ such that $\operatorname{Pos}\left\{\theta_{1 z}\right\} \geq \varepsilon$ where $\varepsilon$ is a sufficiently small number. Thus, a real vector $S\left(\theta_{1 z}\right)$ can be obtained.

Step 3. Calculate

$$
\begin{aligned}
& \frac{S\left(\theta_{1 z}\right)+\sum_{i=1}^{n}\left(s_{i}^{W} / k_{i}\right)}{T}+\sum_{i=1}^{n} \frac{\left(f_{i}-1\right) k_{i} T D_{i} h_{i}^{W}}{2 f_{i}} \\
& +\sum_{i=1}^{n} \frac{f_{i} s_{i}^{R}}{k_{i} T}+\sum_{i=1}^{n} \frac{k_{i} T D_{i} h_{i}^{R}}{2 f_{i}}
\end{aligned}
$$

and $\mu=\mu_{\tilde{S}}\left(S_{1 z}\right)$.
Step 4. If $C\left(T, k_{i}^{\prime} s, f_{i}^{\prime} s\right) \leq \mathrm{TC}$ and $e_{1}<\mu$, set $e_{1}=\mu$; otherwise, if $C\left(T, k_{i}^{\prime} s, f_{i}^{\prime} s\right)>$ TC - and $e_{2}<\mu$, set $e_{2}=\mu$.

Step 5. Set $z=z+1$ and return to Step 2 until the maximum iteration times is reached.

Step 6. Return $e=(1 / 2) *\left(e_{1}+1-e_{2}\right)$, where $e_{1}$ denotes $\operatorname{Pos}\left\{C\left(T, k_{i}^{\prime} s, f_{i}^{\prime} s\right) \leq \mathrm{TC}\right\}$ and $e_{2}$ denotes $\operatorname{Pos}\left\{C\left(T, k_{i}^{\prime} s, f_{i}^{\prime} s\right)>\right.$ TC_ $\}$ or $1-\operatorname{Nec}\left\{C\left(T, k_{i}^{\prime} s, f_{i}^{\prime} s\right) \leq \mathrm{TC}_{-}\right\}$.

3.3. Basic DE and Improved DE (IDE). When the objective functions to be optimized are multimodal or the search spaces are particularly irregular, algorithms need to be highly robust in order to avoid getting stuck at local optimal solution. The advantage of $\mathrm{DE}$ is just to obtain the global optimal solution fairly. In the following, we discuss two DEs.

3.3.1. Basic DE. Basic DE consists of three evolution operators: mutation, crossover, and selection. In mutation operator, $\mathrm{DE}$ uses the differences between randomly selected individuals to generate a trial individual. Then crossover operator is used to produce one offspring which is only accepted if it improves on the fitness of parent individual. The process of choosing individuals is called selection. A brief description of the $\mathrm{DE}$ algorithm is as follows.

Step 1 (initialization and representation)

Initialization. The initial population $\Omega$ is created by assigning random values which lies inside the feasible bound of the decision variable. Each individual is generated by

$$
\begin{gathered}
x_{q j, 0}=\operatorname{rand}[0,1] *\left(x_{j}^{(U)}-x_{j}^{(L)}\right)+x_{j}^{(L)}, \\
q=1,2, \ldots, N P, \quad j=1,2, \ldots, N_{d}, \quad x_{j}^{(L)}<x_{j}<x_{j}^{(U)},
\end{gathered}
$$

where $N P$ is the number of individuals; $N_{d}$ is the dimension of each individual; $x_{j}^{(L)}$ and $x_{j}^{(U)}$ represent the low and upper bound of the $j$ th decision parameter respectively; rand $[0,1]$ is a uniformly distributed random number in range $[0,1]$.

Representation. Now, we will demonstrate how our chromosomes can be decoded to a feasible solution and how each chromosome of population is evaluated. For $n=6$, Figure 3 shows what the chromosomes is composed of.

Assuming $x_{j, \text { min }}$ is the lower bound of $j$ th gene and $x_{j, \max }$ is the upper bound of $j$ th gene, it is obviously that $x_{j, \min }$ is 1 for $k_{i} s$ and $f_{i} s$ and $x_{j, \min }$ is 0 for $T$. It is difficult to give the maximum values for decision variables of the fuzzy M-JRD model because $k_{i}$ and $f_{i}$ influence each other. We consider that RAND algorithm mentioned in Section 3.4 can obtain the optimum solution for the crisp JRD model. So we set $x_{j, \max }$ as values of three times the optimal upper bounds obtained by the RAND_JRD for this fuzzy JRD model following the similar experience of Cha et al. [13]; Wang et al. [15]. 


$$
\begin{array}{|c|c|c|c|c|c|c|c|c|c|c|c|c|}
\hline k_{1} & k_{2} & k_{3} & k_{4} & k_{5} & k_{6} & f_{1} & f_{2} & f_{3} & f_{4} & f_{5} & f_{6} & T \\
\hline
\end{array}
$$

Figure 3: Decoding chromosome.

Step 2 (mutation). For each target individual $x_{q, G}(q=$ $1,2, \ldots, N P$ ) of generation $G$, a mutant individual $v_{q, G+1}$ is generated according to

$$
v_{q, G+1}=x_{r_{1}, G}+F *\left(x_{r_{2}, G}-x_{r_{3}, G}\right), \quad r_{1} \neq r_{2} \neq r_{3}
$$

with randomly chosen integer indexes $r_{1}, r_{2}, r_{3} \in$ $\{1,2, \ldots, N P\}$. Note that indexes have to be different from each other and from the running index. $F$ is called mutation factor between $[0,1]$ which controls the amplification of the differential variation $\left(x_{r_{2}, G}-x_{r_{3}, G}\right)$.

Step 3 (crossover). The basic DE crossover operator implements a discrete recombination of the trial individual $v_{q, G+1}$ and the parent individual $x_{q, G}$ to produce offspring $u_{q, G+1}$. The crossover is implemented as follows:

$$
\begin{aligned}
& u_{q j, G+1} \\
& = \begin{cases}v_{q j, G+1} & \text { if rand }(j) \leq C R \text { or } j=r n b(q) \\
x_{q j, G} & \text { otherwise }\end{cases} \\
& \qquad j=1,2, \ldots, N_{d},
\end{aligned}
$$

where $x_{q j, G}$ refers to the $j$ th element of the individual $x_{q, G} \cdot u_{q j, G+1}$ and $v_{q j, G+1}$ are similarly defined. $\operatorname{rand}(j)$ is the $j$ th evaluation of a uniform random number generator between $[0,1] . r n b(q)$ is a randomly chosen index from $1,2, \ldots, N d$ which ensures that $u_{q, G+1}$ gets at least one parameter from $v_{q, G+1}$. Otherwise, no new parent individual would be produced and the population would not alter. CR is the crossover or recombination rate between $[0,1]$ which has to be determined by the user.

Step 4 (selection). The selection in DE is deterministic and simple. The evaluation function of an offspring is one-to-one competition in the DE. It means the resulting trial individual will only replace the original if it has a lower objective function value. Otherwise, the parent will remain in the next generation

$$
x_{q, G+1}= \begin{cases}u_{q, G+1}, & \text { if } \lambda\left(u_{q, G+1}\right) \geq \lambda\left(x_{q, G}\right) \\ x_{q, G}, & \text { if } \lambda\left(u_{q, G+1}\right)<\lambda\left(x_{q, G}\right)\end{cases}
$$

if $\lambda\left(u_{q, G+1}\right)=\lambda\left(x_{q, G}\right)=0$, and then

$$
x_{q, G+1}=\left\{\begin{array}{rr}
u_{q, G+1}, & \text { if } d_{0}\left(\widetilde{C}\left(u_{q, G+1}, 0\right)\right) \\
& <d_{0}\left(\widetilde{C}\left(x_{q, G}, 0\right)\right) \\
x_{q, G}, & \text { if } d_{0}\left(\widetilde{C}\left(u_{q, G+1}, 0\right)\right) \\
& \geq d_{0}\left(\widetilde{C}\left(x_{q, G}, 0\right)\right),
\end{array}\right.
$$

where $\lambda$ is obtained by (13). This is the same for all variants of the DE. Although the selection pressure is only one, the best individual of the next generation will be at least as fit as the best individual of the current generation.

Step 5 (stop and output results). When stopping criterion is met, output the optimal results; otherwise, repeat Step 2-Step 4. In this study, stopping criterion is met when maximum number of iteration $\left(G_{\max }\right)$ is reached.

3.3.2. The IDE. The key difference between the IDE and basic $\mathrm{DE}$ is in the way of adjusting scale mutation factor $F$ and crossover rate CR. The method modifies mutation factor $F$ according to the objective function values of all candidate solutions in mutation step and adjusts crossover rate CR in terms of iteration number in crossover step. Both modified operators can not only diversify candidate solutions, but also increase the convergence of algorithm. In short, the IDE and $\mathrm{DE}$ are different in two aspects.

(1) For mutation $F$ of DE, it is set to a fixed value for all candidate solutions over all iterations. That is to say, all candidate solutions have the same magnification factor of the differential variation $x_{r_{2}, G}-x_{r_{3}, G}$. Here, if the objective function is minimizing the total cost, an adaptive scale factor $F_{q}$ for the $q$ th candidate solution is stated as follows:

$$
F_{q}= \begin{cases}\frac{f_{q}-f_{\min }}{f_{\text {aver }}-f_{\min }} \times \operatorname{rand}_{k} & \text { if } \frac{f_{q}-f_{\min }}{f_{\text {aver }}-f_{\min }}<2 \\ 2 \times \text { rand }_{q} & \text { otherwise. }\end{cases}
$$

If the objective function is maximizing the credibility, an adaptive scale factor $F_{q}$ for the $q$ th candidate solution is stated as follows:

$$
F_{q}= \begin{cases}\frac{f_{\max }-f_{q}}{f_{\max }-f_{\text {aver }}} \times \operatorname{rand}_{k} & \text { if } \frac{f_{\max }-f_{q}}{f_{\max }-f_{\text {aver }}}<2 \\ 2 \times \text { rand }_{q} & \text { otherwise, }\end{cases}
$$

where rand $_{q}$ belongs to a uniform distribution in the ranges $[0,1] ; f_{q}$ represents the objective value of the $q$ th solution; $f_{\min }$ and $f_{\max }$ represent the minimal and maximal objective function values of all candidate solutions, respectively; $f_{\text {aver }}$ represents the average objective function value of all solutions.

(2) For crossover rate CR of DE, it is set to a fixed value for any dimension of any candidate solution over all iterations. In other words, any dimension of any candidate solution has the same crossover rate, which does not change with the evolution process. Here, a dynamic crossover $\mathrm{CR}_{G}$ is adopted for all candidate solutions at iteration $G$, and it is stated at follows:

$$
\begin{gathered}
\mathrm{CR}_{G}=a \times \exp \left(b \times G^{2}\right), \\
b=\frac{1}{G_{\max }^{2}-1} \ln \left(\frac{\mathrm{CR}_{\text {max }}}{\mathrm{CR}_{\text {min }}}\right), \quad a=\mathrm{CR}_{\text {min }} \exp (-b),
\end{gathered}
$$


where $G$ and $G_{\max }$ are current iteration number and maximal iteration number, respectively; $\mathrm{CR}_{\min }$ and $\mathrm{CR}_{\max }$ represent minimal crossover rate and maximal crossover, respectively.

\subsection{Modified Algorithm for the Defuzzified JRD Model}

(1) Optimal T. For a given set of $k_{i} s$ and $f_{i} s$, the optimal basic cycle time $T$ can be easily obtained as shown in (23) from the first order derivative of the defuzzified TC function since the $\mathrm{TC}$ function is convex in $T$ :

$$
T^{*}=\sqrt{\frac{2\left(((\underline{S}+2 S+\bar{S}) / 4)+\sum_{i=1}^{n}\left(\left(s_{i}^{W}+f_{i} s_{i}^{R}\right) / k_{i}\right)\right)}{\sum_{i=1}^{n} k_{i} D_{i}\left(h_{i}^{W}+\left(\left(h_{i}^{R}-h_{i}^{W}\right) / f_{i}\right)\right)}} .
$$

(2) Optimal $k_{i}$ and $f_{i}$. According to experiences of $[13-15,17]$, for a given set of $f_{i} s$ and $T$, the optimality condition of $k_{i}$ can be derived from the following two conditions:

$$
\begin{aligned}
& d\left(\widetilde{C}, 0, k_{i}\right) \leq d\left(\widetilde{C}, 0, k_{i}+1\right), \\
& d\left(\widetilde{C}, 0, k_{i}\right) \leq\left(\widetilde{C}, 0, k_{i}-1\right) .
\end{aligned}
$$
follows:

Therefore, the optimality condition of $k_{i}$ is defined as

$$
k_{i}\left(k_{i}-1\right) \leq \frac{2\left(s_{i}^{W}+f_{i} s_{i}^{R}\right)}{T^{2} D_{i}\left(h_{i}^{W}+\left(\left(h_{i}^{R}-h_{i}^{W}\right) / f_{i}\right)\right)} \leq k_{i}\left(k_{i}+1\right) .
$$

Similarly, the optimality condition of $f_{i}$ is defined as follows:

$$
f_{i}\left(f_{i}-1\right) \leq \frac{k_{i}^{2} T^{2} D_{i}\left(h_{i}^{R}-h_{i}^{W}\right)}{2 s_{i}^{R}} \leq f_{i}\left(f_{i}+1\right) .
$$

(3) RAND_JRD: A Modified Algorithm for the Defuzzified JRD. Cha et al. [13] proposed a modified RAND algorithm for the crisp JRD model to minimize the TC. Making corresponding changes in $T_{\max }$, an algorithm named RAND_JRD can be used to find the optimal solution. The procedures are as follows:

$\begin{array}{lccc}\text { Step } 1 . \quad \text { Compute } & T_{\max } & = \\ \sqrt{2\left(((\underline{S}+2 S+\bar{S}) / 4)+\sum_{i=1}^{n} s_{i}\right) / \sum_{i=1}^{n} D_{i} h_{i}} & \text { and } T_{\min } & =\end{array}$ $\min \sqrt{2 s_{i} / D_{i} h_{i}}$ for each $i$.

Step 2. Divide the range $\left[T_{\max }, T_{\min }\right]$ into $m_{1}$ different equally spaced values of $T\left(T_{1}, \ldots, T_{j_{1}}, \ldots, T_{m 1}\right)$. The value of $m_{1}$ is decided by the decision-maker. Set $j_{-} 1=0$.

Step 3. Set $j_{-} 1=j_{-} 1+1$ and $r=0$. Put $T_{j_{-} 1}(r)=T_{j_{-} 1}$ and $\left(f_{1}(r), f_{2}(r), \ldots, f_{n}(r)\right)=1$.

Step 4. Set $r=r+1$.
Step 5. For a given value of $T_{j_{-1}}(r-1)$ and a given set of $f_{j_{1} 1}(r-1) s$, find the optimal values of $k_{i}$ using (25). Put $k_{i}(r)=k_{i}$.

Step 6. For a given value of $T_{j_{-1}}(r-1)$ and a given set of $k_{i}(r) s$, find the optimal values of $f_{i}$ using (26). Put $f_{i}(r)=f_{i}$.

Step 7. For a given set of $k_{i}(r)$ s and $f_{i}(r)$ s, find the optimal value of $T$ using (23). Put $T_{j_{-1}}(r)=T_{j_{-1}}$.

Step 8. If $T_{j_{-1}}(r) \neq T_{j_{-1}}(r-1)$, go to Step 4. Otherwise, put $T_{j_{-1}}^{*}=T_{j_{1} 1}(r), k_{i j_{1} 1}^{*}=k_{i}(r)$, and $f_{i j_{-1}}^{*}=f_{i}(r)$. Compute $\mathrm{TC}_{j_{-1}}$ for this $\left(T_{j_{-1}}^{*}, k_{i j_{-} 1}^{*} s, f_{i j_{-} 1}^{*} s\right)$.

Step 9. If $j_{-} 1 \neq m_{1}$, go to Step 3 .

Otherwise, stop and select $\left(T_{j_{-1}}^{*}, k_{i j_{1}}^{*} s, f_{i j_{1}}^{*} s\right)$ with the minimum $d_{0}(\widetilde{C}, 0)$.

3.5. Flowchart of Proposed Algorithm for Fuzzy M-JRD Model. Main flowchart of the proposed algorithm is shown in Figure 4 . In the adjustment, if $x_{q j, G}>x_{j, \max }$ or $x_{q j, G}<x_{j, \min }$, then $x_{q j, G}=x_{j, \min }+\operatorname{rand}(1) *\left(x_{j, \max }-x_{j, \min }\right)$, where $\operatorname{rand}(1) \epsilon$ $(0,1)$.

\section{Numerical Examples}

4.1. Experiment 1. Basic Experiment 1 is given to compare the DE and IDE. According to the recommendation of the inventor of DE [37] and the similar experiences of $[4,15,17]$, the factors setting for DE used in all experiments are listed as follows: the population $N P=50$, the mutation factor $F=1.6$, the crossover factor $\mathrm{CR}=0.7$, and the maximum generation is set to 500. Factors of IDE are set as follows: $\mathrm{CR}_{\max }=$ $0.9, \mathrm{CR}_{\min }=0.4$. The data and results of this experiment are shown in Tables 1 and 2. Convergent curves of DE and IDE are given in Figure 5.

We can calculate $d_{0}(\widetilde{C}, 0)^{0}=4694.1, d_{0}(\widetilde{C}, 0)^{1}=5110.1$, $\operatorname{Cr}\{\cdot\}^{0}=0$, and $\operatorname{Cr}\{\cdot\}^{1}=1$.

Then, DE and IDE are performed 50 times and the results are reported in Table 3.

From Table 2, the radios of the achievement levels gained by DE and IDE can be computed as follows:

$$
\begin{gathered}
\mu_{1}=\frac{d_{0}(\widetilde{C}, 0)^{1}-d_{0}(\widetilde{C}, 0)}{d_{0}(\widetilde{C}, 0)^{1}-d_{0}(\widetilde{C}, 0)^{0}}=\frac{5110.1-4831.7}{5110.1-4694.1}=0.6685, \\
\mu_{2}=\frac{\operatorname{Cr}\{\cdot\}-\operatorname{Cr}\{\cdot\}^{0}}{\operatorname{Cr}\{\cdot\}^{1}-\operatorname{Cr}\{\cdot\}^{0}}=\frac{0.8417-0}{1-0}=0.8417 ; \\
\frac{\mu_{1}}{\mu_{2}}=\frac{0.6685}{0.8417}=0.794 \approx \frac{w_{1}}{w_{2}}=\frac{0.44}{0.56}=0.785 .
\end{gathered}
$$

Results in Tables 2 and 3 show (1) the ratios of the achievement levels and the weights for the fuzzy objectives are nearly equivalent using IWFNLP and DE/IDE; (2) IDE outperforms DE with a rapid convergence speed. 


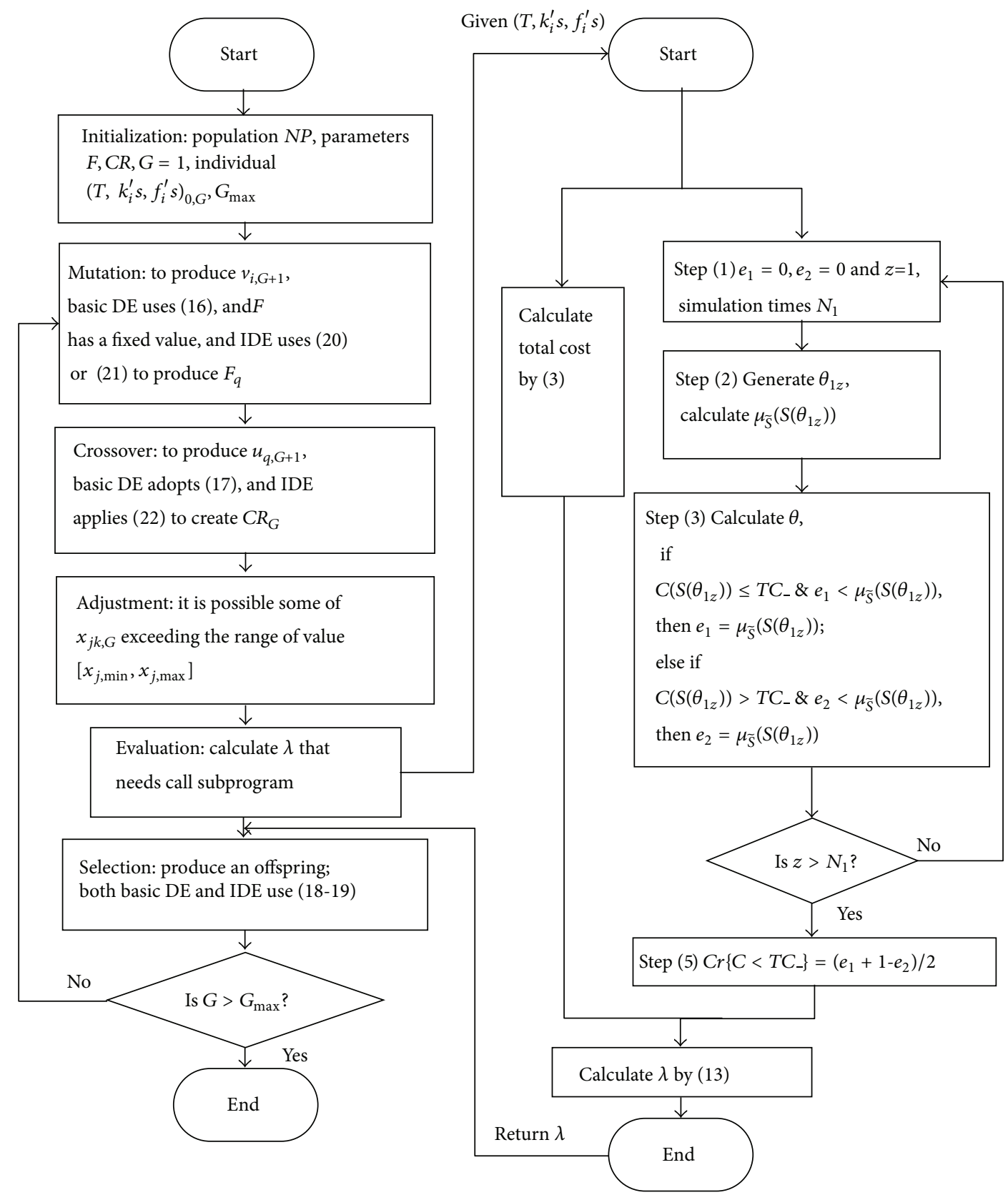

FIGURE 4: Main flowchart of the proposed algorithm.

4.2. Experiment 2 with Different Major Ordering Cost. Khouja and Goyal [9] indicated that the value of major ordering cost affected the performance of algorithms. So three examples were designed to test the IDE. The data are same for $D_{i}, s_{i}^{W}$, $h_{i}^{W}, h_{i}^{R}, h_{i}^{R}, w_{1}$, and $w_{2}$ as shown in Table 2 . But the values of $\widetilde{S}$ are different as shown in Table 4 . The results are listed in Table 5. Figure 6 shows the convergent processes.

From Table 5 and Figure 6, we can conclude that the IDE can also find satisfactory solutions with different values of major ordering cost. The ratios of the achievement levels and the weights for the fuzzy objectives are still nearly equivalent using IWFNLP method and IDE.
TABLE 1: Data for Experiment 1.

\begin{tabular}{lcccccc}
\hline Item $i$ & 1 & 2 & 3 & 4 & 5 & 6 \\
\hline$D_{i}$ & 10,000 & 5,000 & 3,000 & 1,000 & 600 & 200 \\
$s_{i}^{W}$ & 45 & 46 & 47 & 44 & 45 & 47 \\
$h_{i}^{W}$ & 1 & 1 & 1 & 1 & 1 & 1 \\
$s_{i}^{R}$ & 5 & 5 & 5 & 5 & 5 & 5 \\
$h_{i}^{R}$ & 1.5 & 1.5 & 1.5 & 1.5 & 1.5 & 1.5 \\
\hline$\widetilde{S}$ & \multicolumn{3}{c}{$(175,185,255)$} & & TC & \multicolumn{2}{c}{5000} \\
\hline$w_{1}$ & 0.44 & & $w_{2}$ & \multicolumn{2}{c}{0.56} \\
\hline
\end{tabular}




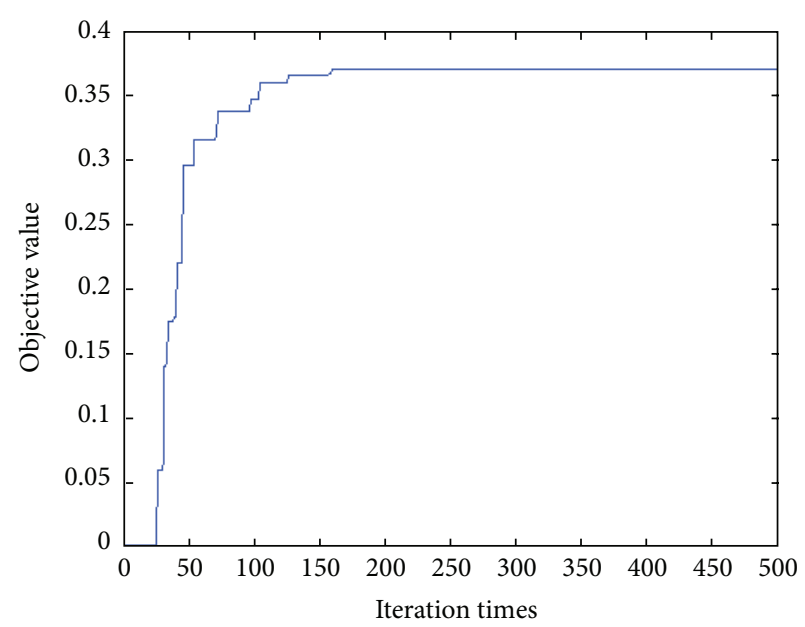

(a) Convergent curve of DE

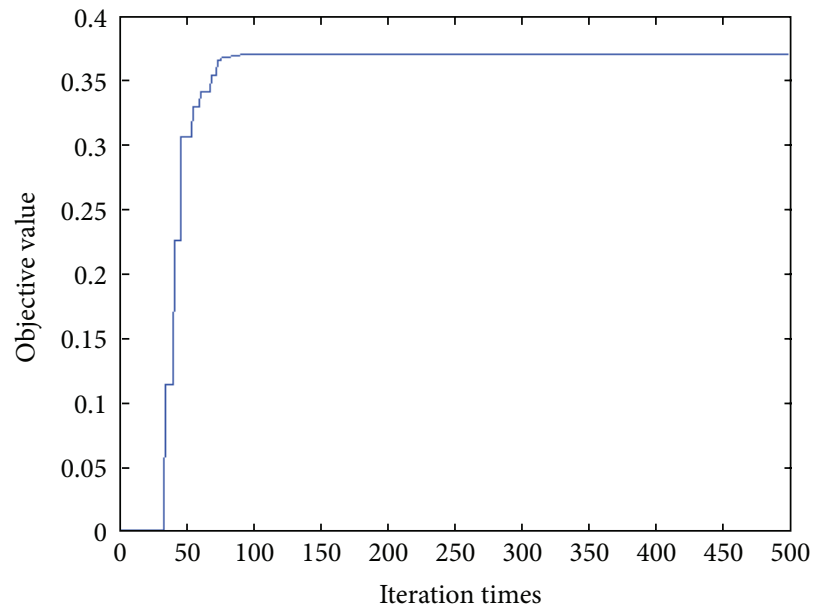

(b) Convergent curve of IDE

FIGURE 5: Convergent curve of DE and IDE.

TABLE 2: The results for Experiment 1.

\begin{tabular}{lcccccc}
\hline Algorithm & $T$ & $k_{i}$ & $f_{i}$ & $\lambda$ & $d(\widetilde{C}, 0)$ & $C r\left\{\widetilde{C}\left(T, k_{i}^{\prime} s, f_{i}^{\prime} s\right) \leq \mathrm{TC}\right\}$ \\
\hline DE & 0.195 & $(1,1,1,2,2,4)$ & $(4,3,2,3,2,2)$ & 0.341 & 4831.7 & 0.8417 \\
IDE & 0.195 & $(1,1,1,2,2,4)$ & $(4,3,2,3,2,2)$ & 0.341 & 4831.7 & 0.8417 \\
\hline
\end{tabular}

TABLE 3: Comparison of DE and IDE.

\begin{tabular}{lcccccc}
\hline Algorithm & Convergence times & $\begin{array}{c}\text { Average iteration } \\
\text { times }\end{array}$ & $\begin{array}{c}\text { Maximum iteration } \\
\text { times }\end{array}$ & $\begin{array}{c}\text { Minimum iteration } \\
\text { times }\end{array}$ & Average $\lambda$ & Minimum $\lambda$ \\
\hline $\mathrm{DE}$ & 50 & 234.88 & 283 & 180 & 0.3704 \\
$\mathrm{IDE}$ & 50 & 85.66 & 104 & 68 & 0.3704 \\
\hline
\end{tabular}

TABLE 4: Data for Experiments 2a, 2b, and 2c.

\begin{tabular}{llcll}
\hline Experiment 2a & $\widetilde{S}$ & $(4,10,16)$ & TC & 3639 \\
Experiment 2b & $\widetilde{S}$ & $(38,45,52)$ & TC & 3912 \\
Experiment 2c & $\widetilde{S}$ & $(1000,1060,1280)$ & TC & 8267 \\
\hline
\end{tabular}

\section{Conclusions and Future Research}

This paper is an interdisciplinary research of the fuzzy inventory model and intelligent optimization algorithm. Due to the inevitable uncertainty, it is quite natural for decisionmaker to assume two main goals: (1) nonrigid total cost goal; (2) credibility goal to assure the safety of cash flow. We developed a practical JRD model under uncertainty and provided an effective algorithm for this model. The main contributions are as follows.

(1) Actually, there are lots of papers discussed inventory and risk management issues [45]. However, to our best knowledge, the JRD model under fuzzy costs that simultaneously minimizes the total cost and maximizes the credibility to assure the safety of cash flow is nonexistence. Our work provides a useful approach for the joint replenishment and delivery scheduling under uncertainty.
(2) The formulation of the proposed M-JRD model is handled by the IWFNLP which can make the ratios of the achievement levels of objectives and the weights for the fuzzy objectives are nearly equivalent. The IWFNLP method gives the solution that satisfies the decision-maker's desirable achievement level of the total cost objective and credibility objective. It is an effective decision tool to ensure a decisionmaker's expectation is achieved.

(3) Hybrid intelligent algorithms are designed to solve the proposed JRD handled by the IWFNLP method using the FSA and DE/IDE. Results of numerical examples show the IDE can find satisfactory solutions faster than DE.

Other intelligent algorithms, such as genetic-simulated annealing algorithm [46] and quantum evolution algorithm [47], also show good performances to solve complex optimization problems. In the future, we will design hybrid algorithms by taking the advantages of the above algorithms to handle more complex fuzzy JRD problems. Moreover, another future research direction is to optimize the fuzzy JRD problem under different operational risks because of the inevitable uncertainty under supply chain environment for enterprises in the network economic era [48-50]. 
TABLE 5: Results for Experiment 2 using IDE.

\begin{tabular}{lcccccccc}
\hline Experiment number & $T$ & $k_{i}$ & $f_{i}$ & $\lambda$ & $d(\widetilde{C}, 0)$ & $C r\{\cdot\}$ & $\boldsymbol{\mu}_{\mathbf{1}} / \boldsymbol{\mu}_{\mathbf{2}}$ & $\mathbf{w}_{\mathbf{1}} / \mathbf{w}_{\mathbf{2}}$ \\
\hline 2a & 0.1066 & $(1,1,2,3,4,6)$ & $(2,2,3,2,2,2)$ & 0.2745 & 3625.1 & 0.6240 & $\mathbf{0 . 7 8 5 4}$ & $\mathbf{0 . 7 8 5 7}$ \\
2b & 0.1410 & $(1,1,1,2,3,5)$ & $(3,2,2,2,2,2)$ & 0.2781 & 3899.0 & 0.6320 & $\mathbf{0 . 7 8 6 1}$ & $\mathbf{0 . 7 8 5 7}$ \\
2c & 0.3683 & $(1,1,1,1,1,2)$ & $(8,6,5,3,2,2)$ & 0.3534 & 8013.0 & 0.8033 & $\mathbf{0 . 7 8 5 5}$ & $\mathbf{0 . 7 8 5 7}$ \\
\hline
\end{tabular}

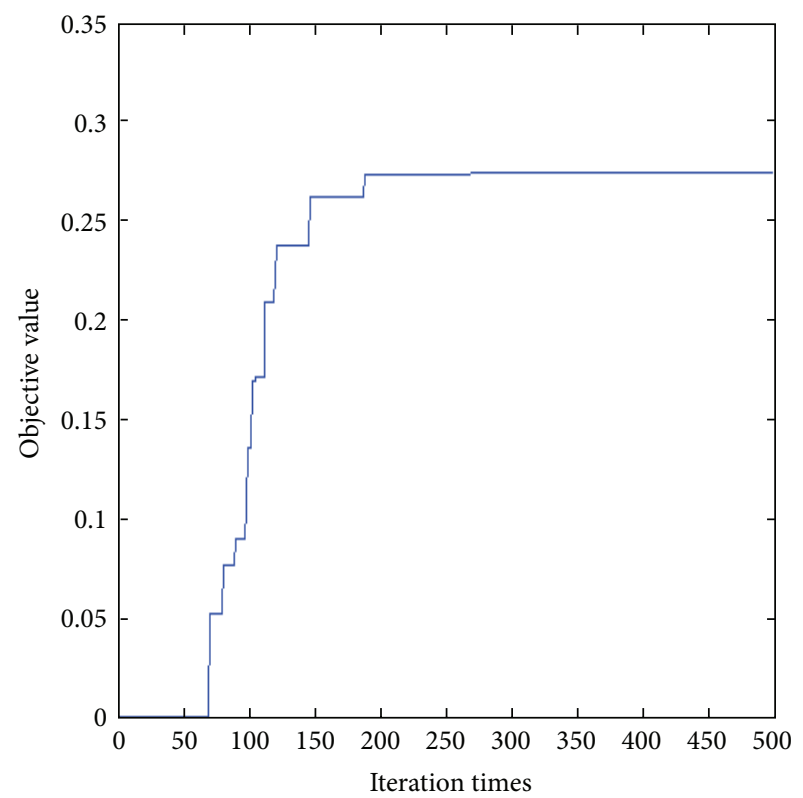

(a) Experiment 2a

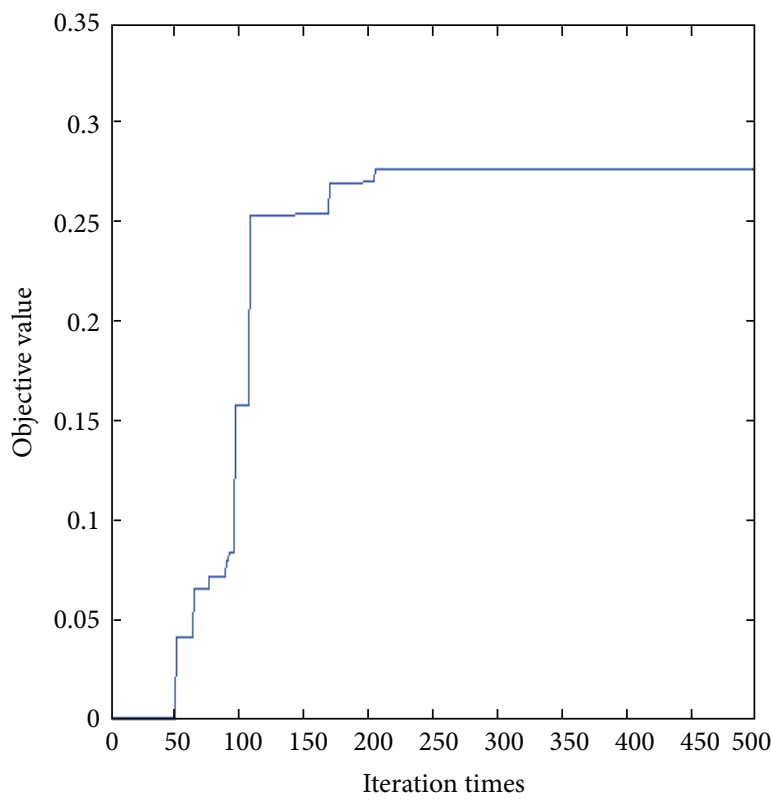

(b) Experiment $2 \mathrm{~b}$

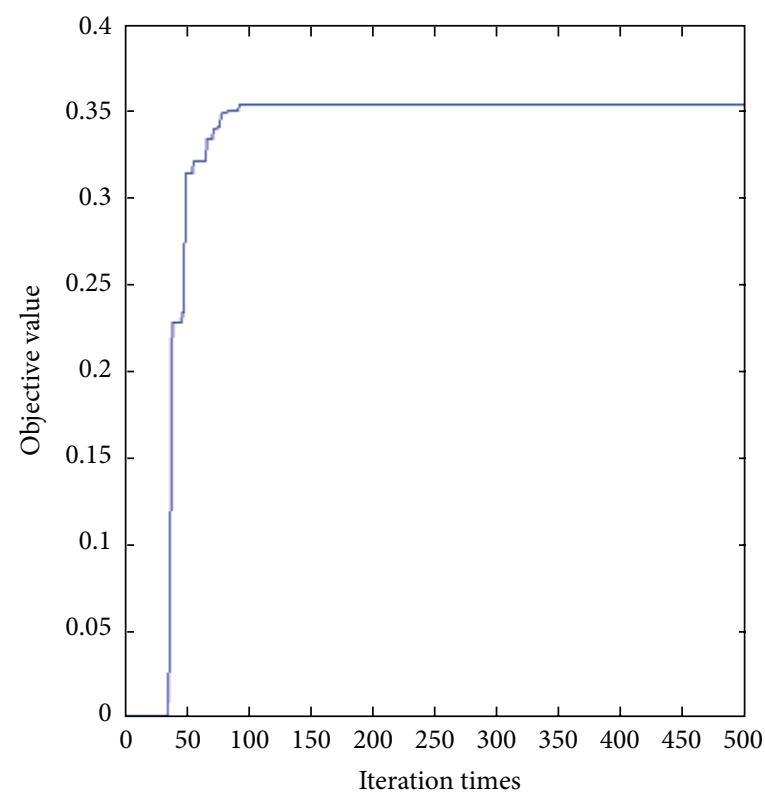

(c) Experiment 2c

FIGURE 6: Convergent curve of IDE for Experiment 2. 


\section{Conflict of Interests}

The authors declare that there is no conflict of interests regarding the publication of this paper.

\section{Acknowledgments}

This research is partially supported by National Natural Science Foundation of China (nos. 71371080 and 71131004), Humanities and Social Sciences Foundation of Chinese Ministry of Education (no. 11YJC630275), and Fundamental Research Funds for the Central Universities (HUST: 2014QN201).

\section{References}

[1] S.-L. Hsu, "Optimal joint replenishment decisions for a central factory with multiple satellite factories," Expert Systems with Applications, vol. 36, no. 2, pp. 2494-2502, 2009.

[2] S. K. Goyal, "Determination of optimum packaging frequency of items jointly replenished," Management Science, vol. 21, no. 4, pp. 436-443, 1974.

[3] L. Wang, J. He, and Y. Zeng, "A differential evolution algorithm for joint replenishment problem using direct grouping and its application," Expert Systems, vol. 29, no. 5, pp. 429-441, 2012.

[4] L. Wang, J. He, D. Wu, and Y. Zeng, "A novel differential evolution algorithm for joint replenishment problem under interdependence and its application," International Journal of Production Economics, vol. 135, no. 1, pp. 190-198, 2012.

[5] M. Kaspi and M. J. Rosenblatt, "On the economic ordering quantity for jointly replenished items," International Journal of Production Research, vol. 29, no. 1, pp. 107-114, 1991.

[6] A. L. Olsen, "An evolutionary algorithm to solve the joint replenishment problem using direct grouping," Computers and Industrial Engineering, vol. 48, no. 2, pp. 223-235, 2005.

[7] F. C. Lee and M. J. Yao, "A global optimum search algorithm for the joint replenishment problem under power-of-two policy," Computers and Operations Research, vol. 30, no. 9, pp. 1319-1333, 2003.

[8] E. Porras and R. Dekker, "A solution method for the joint replenishment problem with correction factor," International Journal of Production Economics, vol. 113, no. 2, pp. 834-851, 2008.

[9] M. Khouja and S. Goyal, "A review of the joint replenishment problem literature: 1989-2005," European Journal of Operational Research, vol. 186, no. 1, pp. 1-16, 2008.

[10] A. Narayanan, P. Robinson, and F. Sahin, "Coordinated deterministic dynamic demand lot-sizing problem: a review of models and algorithms," Omega, vol. 37, no. 1, pp. 3-15, 2009.

[11] S. Sindhuchao, H. E. Romeijn, E. Akçali, and R. Boondiskulchok, "An integrated inventory-routing system for multi-item joint replenishment with limited vehicle capacity," Journal of Global Optimization, vol. 32, no. 1, pp. 93-118, 2005.

[12] C. K. Chan, L. Y. Li, C. T. Ng, B. K. Cheung, and A. Langevin, "Scheduling of multi-buyer joint replenishments," International Journal of Production Economics, vol. 102, no. 1, pp. 132-142, 2006.

[13] B. C. Cha, I. K. Moon, and J. H. Park, "The joint replenishment and delivery scheduling of the one-warehouse, n-retailer system," Transportation Research E: Logistics and Transportation Review, vol. 44, no. 5, pp. 720-730, 2008.
[14] I. K. Moon, B. C. Cha, and C. U. Lee, "The joint replenishment and freight consolidation of a warehouse in a supply chain," International Journal of Production Economics, vol. 133, no. 1, pp. 344-350, 2011.

[15] L. Wang, C.-X. Dun, W.-J. Bi, and Y.-R. Zeng, "An effective and efficient differential evolution algorithm for the integrated stochastic joint replenishment and delivery model," KnowledgeBased Systems, vol. 36, pp. 104-114, 2012.

[16] W. W. Qu, J. H. Bookbinder, and P. Iyogun, "An integrated inventory-transportation system with modified periodic policy for multiple products," European Journal of Operational Research, vol. 115, no. 2, pp. 254-269, 1999.

[17] L. Wang, H. Qu, S. Liu, and C. X. Dun, "Modeling and optimization of the multiobjective stochastic joint replenishment and delivery problem under supply chain environment," The Scientific World Journal, vol. 2013, Article ID 916057, 11 pages, 2013.

[18] L. G. Cui, L. Wang, and J. Deng, "RFID technology investment evaluation model for the stochastic joint replenishment and delivery problem," Expert Systems with Applications, vol. 41, no. 4, pp. 1792-1805, 2014.

[19] M. S. Pishvaee and S. A. Torabi, "A possibilistic programming approach for closed-loop supply chain network design under uncertainty," Fuzzy Sets and Systems, vol. 161, no. 20, pp. 2668$2683,2010$.

[20] Y. Zeng, L. Wang, and J. He, "A novel approach for evaluating control criticality of spare parts using fuzzy comprehensive evaluation and GRA," International Journal of Fuzzy Systems, vol. 14, no. 3, pp. 392-401, 2012.

[21] J. Chiang, J. S. Yao, and H. M. Lee, "Fuzzy inventory with backorder defuzzification by signed distance method," Journal of Information Science and Engineering, vol. 21, no. 4, pp. 673694, 2005.

[22] J. Kacprzyk and P. Stanieski, "Long-term inventory policymaking through fuzzy decision-making models," Fuzzy Sets and Systems, vol. 8, no. 2, pp. 117-132, 1982.

[23] L. Wang, H. Qu, Y. H. Li, and J. He, "Modeling and optimization of stochastic joint replenishment and delivery scheduling problem with uncertain costs," Discrete Dynamics in Nature and Society, vol. 2013, Article ID 657465, 12 pages, 2013.

[24] L. Wang, C. Dun, C. Lee, Q. Fu, and Y. Zeng, "Model and algorithm for fuzzy joint replenishment and delivery scheduling without explicit membership function," International Journal of Advanced Manufacturing Technology, vol. 66, no. 9-12, pp. 19071920, 2013.

[25] H. Wee, C. Lo, and P. Hsu, "A multi-objective joint replenishment inventory model of deteriorated items in a fuzzy environment," European Journal of Operational Research, vol. 197, no. 2, pp. 620-631, 2009.

[26] A. Roy, S. Kar, and M. Maiti, "A deteriorating multi-item inventory model with fuzzy costs and resources based on two different defuzzification techniques," Applied Mathematical Modelling, vol. 32, no. 2, pp. 208-223, 2008.

[27] R. Handfield, D. Warsing, and X. M. Wu, " $(Q, r)$ inventory policies in a fuzzy uncertain supply chain environment," European Journal of Operational Research, vol. 197, no. 2, pp. 609-619, 2009.

[28] B. D. Liu, "Dependent-chance programming in fuzzy environments," Fuzzy Sets and Systems, vol. 109, no. 1, pp. 97-106, 2000.

[29] L. Wang, Q.-L. Fu, C.-G. Lee, and Y.-R. Zeng, "Model and algorithm of fuzzy joint replenishment problem under credibility 
measure on fuzzy goal," Knowledge-Based Systems, vol. 39, pp. 57-66, 2013.

[30] H. Peng and H. Zhou, "A fuzzy-dependent chance multiobjective programming for water resources planning of a coastal city under fuzzy environment," Water and Environment Journal, vol. 25, no. 1, pp. 40-54, 2011.

[31] L. Lu and M. E. Posner, "Approximation procedures for the onewarehouse multi-retailer system," Management Science, vol. 40, no. 10, pp. 1305-1316, 1994.

[32] R. Wang and L. Wang, "Maximum cut in fuzzy nature: models and algorithms," Journal of Computational and Applied Mathematics, vol. 234, no. 1, pp. 240-252, 2010.

[33] X. B. Wang, W. S. Tang, and R. Q. Zhao, "Fuzzy economic order quantity inventory models without backordering," Tsinghua Science and Technology, vol. 12, no. 1, pp. 91-96, 2007.

[34] R. Narasimhan, "Goal programming in a fuzzy environment," Decision Science, vol. 11, no. 2, pp. 325-336, 1980.

[35] L. H. Chen and F. C. Tsai, "Fuzzy goal programming with different importance and priorities," European Journal of Operational Research, vol. 133, no. 3, pp. 548-556, 2001.

[36] H. Ke and B. Liu, "Fuzzy project scheduling problem and its hybrid intelligent algorithm," Applied Mathematical Modelling: Simulation and Computation for Engineering and Environmental Systems, vol. 34, no. 2, pp. 301-308, 2010.

[37] R. Storn and K. Price, "Differential evolution-a simple and efficient heuristic for global optimization over continuous spaces," Journal of Global Optimization, vol. 11, no. 4, pp. 341359, 1997.

[38] L. Wang, Q. Fu, and Y. Zeng, "Continuous review inventory models with a mixture of backorders and lost sales under fuzzy demand and different decision situations," Expert Systems with Applications, vol. 39, no. 4, pp. 4181-4189, 2012.

[39] H. Qu, L. Wang, and Y. R. Zeng, "Modeling and optimization for the joint replenishment and delivery problem with heterogeneous items," Knowledge-Based Systems, vol. 54, pp. 207-215, 2013.

[40] L. Wang, H. Qu, T. Chen, and F. P. Yan, "An effective hybrid self-adapting differential evolution algorithm for the joint replenishment and location-inventory problem in a three-level supply chain," The Scientific World Journal, vol. 2013, Article ID 270249, 11 pages, 2013.

[41] F. Al-Obeidat, N. Belacel, J. A. Carretero, and P. Mahanti, "Differential Evolution for learning the classification method PROAFTN," Knowledge-Based Systems, vol. 23, no. 5, pp. 418426, 2010.

[42] L. Wang, H. Qu, S. Liu, and C. Chen, "Optimizing the joint replenishment and channel coordination problem under supply chain environment using a simple and effective differential evolution algorithm," Discrete Dynamics in Nature and Society, vol. 2014, Article ID 709856, 12 pages, 2014.

[43] Q. K. Pan and L. Wang, "A novel differential evolution algorithm for no-idle permutation flow-shop scheduling problems," European Journal of Industrial Engineering, vol. 2, no. 3, pp. 279-297, 2008.

[44] D. Zou, H. Liu, L. Gao, and S. Li, "An improved differential evolution algorithm for the task assignment problem," Engineering Applications of Artificial Intelligence, vol. 24, no. 4, pp. 616-624, 2011.

[45] Y. M. Gong, Global Operations Strategy: Fundamentals and Practice, Springer, 2013.
[46] Y. H. Li, H. Guo, L. Wang, and J. Fu, "A hybrid geneticsimulated annealing algorithm for the location-inventory-routing problem considering returns under esupply chain environment," The Scientific World Journal, vol. 2013, Article ID 125893, 10 pages, 2013.

[47] L. Cui, L. Wang, J. Deng, and J. Zhang, "A new improved quantum evolution algorithm with local search procedure for capacitated vehicle routing problem," Mathematical Problems in Engineering, vol. 2013, Article ID 159495, 17 pages, 2013.

[48] S. Liu and L. Wang, "Understanding the impact of risks on performance in internal and outsourced information technology projects: the role of strategic importance," International Journal of Project Management, 2014.

[49] H. Soleimani, M. Seyyed-Esfahani, and G. Kannan, "Incorporating risk measures in closed-loop supply chain network design," International Journal of Production Research, vol. 52, no. 6, pp. 1843-1867, 2014.

[50] S. Liu and L. Wang, "User liaisons perspective on behavior and outcome control in IT projects: role of IT experience, behavior observability, and outcome measurability," Management Decision, vol. 52, no. 6, pp. 1148-1173, 2014. 


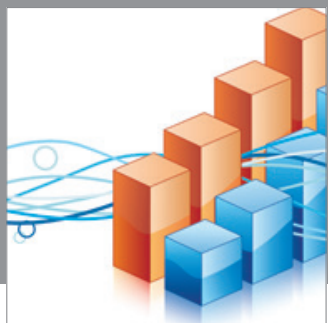

Advances in

Operations Research

mansans

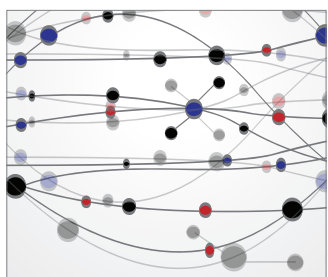

The Scientific World Journal
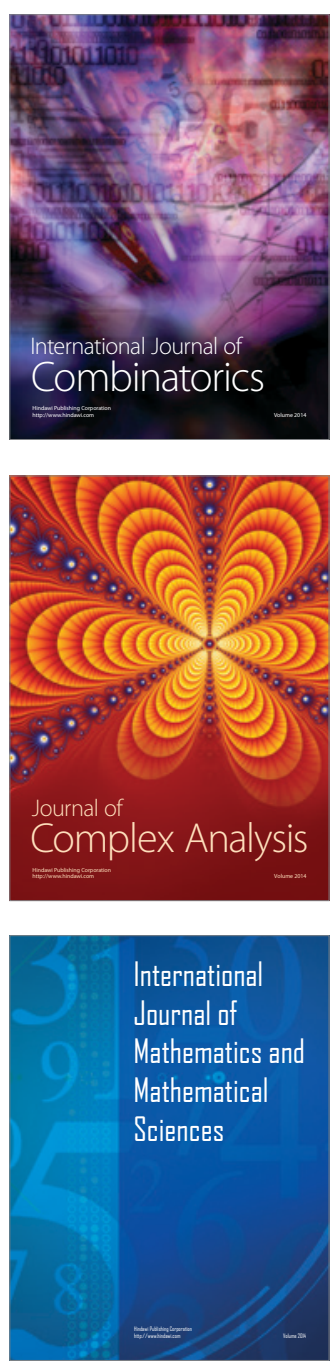
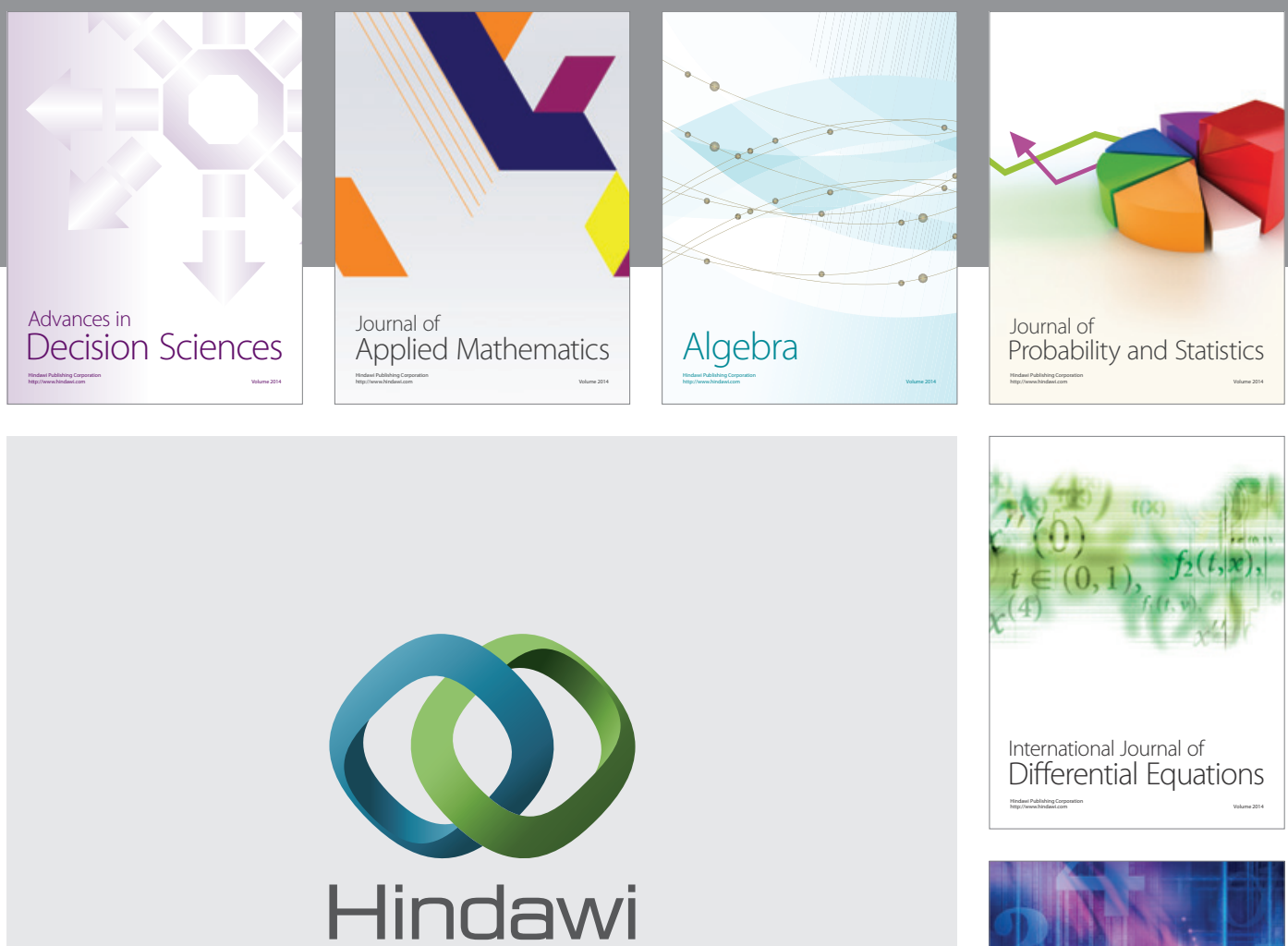

Submit your manuscripts at http://www.hindawi.com
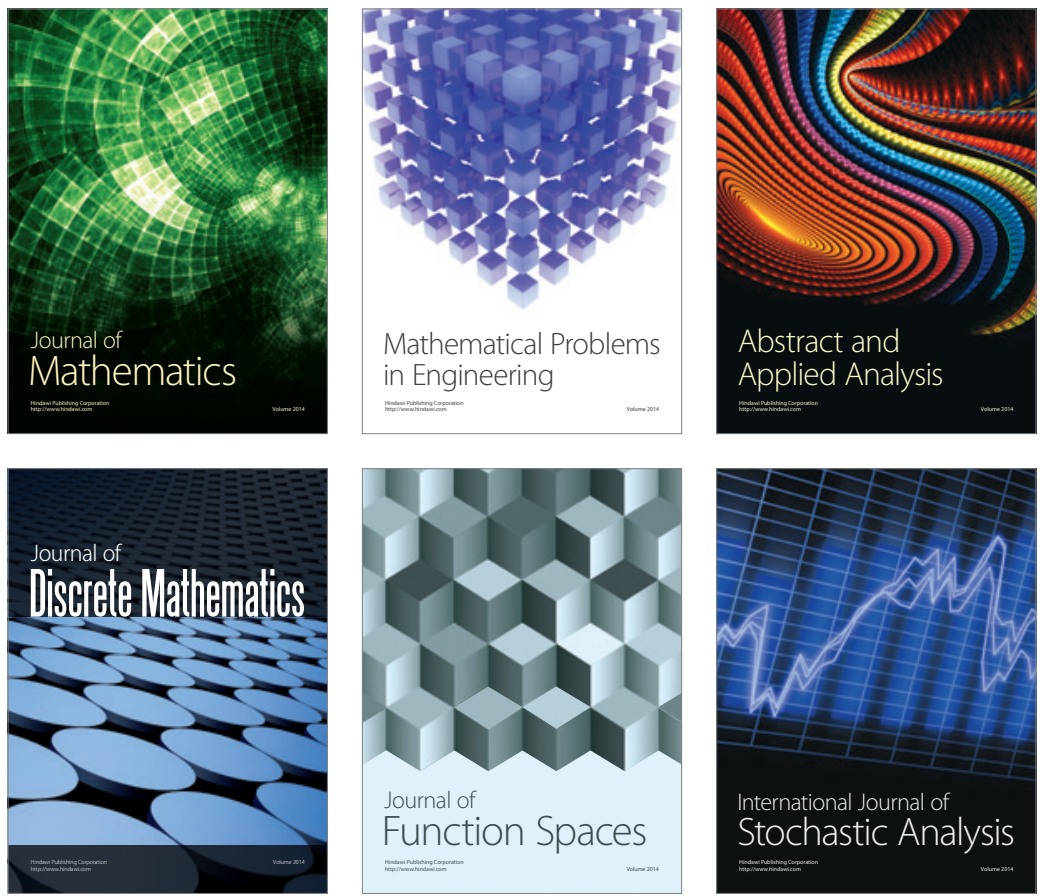

Journal of

Function Spaces

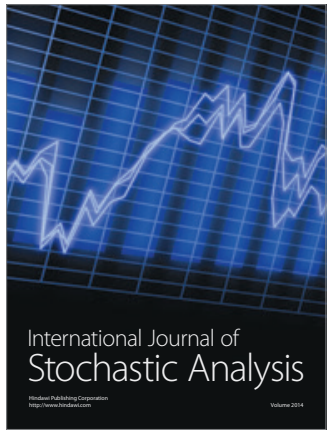

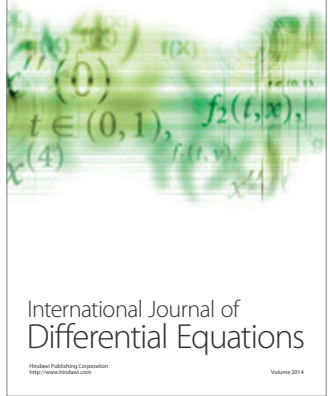
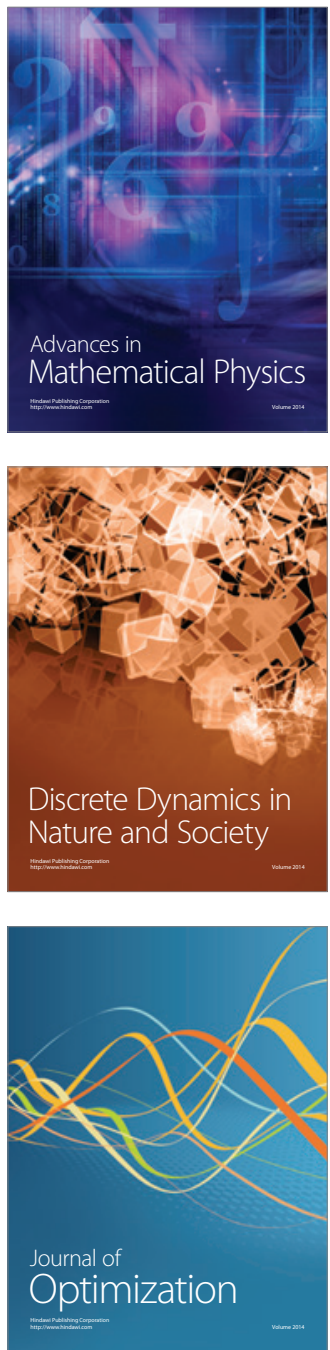